\title{
A Generally Applicable Laser Doppler Velocimetry
}

\author{
Zhao Lili ${ }^{\mathrm{a}}$, Jin Meishan ${ }^{\mathrm{b}}$, Li Jing ${ }^{\mathrm{c}}$ \\ Department of Electrical and Electronic, Aviation University of Airforce, Changchun,130022, China \\ aemail: cacique2000@sina.com, ${ }^{b}$ email: \\ Jinmeishan2014@163.com, \\ cemail: lijingemail2014@yeah.net
}

Keywords: Doppler Effect; Velocimetry; Beating Frequency; Short Time Fourier Transform

\begin{abstract}
Photonic Doppler velocimetry (PDV) is a fiber-based interferometer which has been widely used in dynamic shock physics research. Although the conventional PDV systems are simple to construct, it has special requirement to the probe for getting appropriate amount of reference light. This paper proposes to change the way in which getting the reference light to construct a generally applicable photon Doppler velocimetry system. Short time Fourier transform is utilized in order to extract frequency information from the raw data recorded by the digitizer.
\end{abstract}

\section{Introduction}

In the 1960's the laser based velocimetry started to be used in the flow velocity measurement of the gas and liquid [1][2][3]. Then it has been improved considerably over the decades and formed the diagnostic known as VISAR which represents for velocity interferometer system for any reflector [4][5]. In the typical VISAR system, Doppler shifted light from a moving target is split along two different paths: a direct leg and a delay leg. The altered frequency interacts with the other frequency and produce a beating frequency. While the traditional VISAR system is expensive and rather complicated in construction and maintenance because of the path of light is constituted by discrete optical element. The interferometric fiber-optic Doppler velocimeter has been researched from 1980's and has made great development. In 1996, AFVISAR (All-fiber VISAR) was used to measure velocity for the first time by Levin from Israel [6]. In the AFVISAR system, the optic fiber was used to replace the optic elements in the traditional VISAR system. The fiber-optic Doppler velocimeter is evolved from the laser Doppler velocimeter (LDV) and has proved to be smaller, lighter and simpler in the structure. In 2005, Strand and his colleagues at LLNL developed a laser based velocimetry named PDV (Photonic Doppler Velocimeter) which employs mainly off-the-shelf components developed for the telecommunications industry. Developments in telecom technologies have enabled PDV the advantages of compact, flexible arrangement, easy-to-use and relatively inexpensive compared to the traditional Doppler laser velocimetry [7].

In the PDV concept, the reference laser beam at original frequency is sent along a fiber optic probe that also collects the reflected. Then the returned light obtained from the back-reflectance of the probe interact with a portion of original laser light. If the target is moving, the difference in frequencies produces interference. Then the velocity of the surface can be calculated from the beat frequency. The beat frequency is proportional to the velocity of the moving target and is given by

$$
\Delta f=2 v / \lambda
$$

where $\lambda$ is the wavelength of laser. The beat frequency is temporally recorded with a high speed digitizer. Then the data should be converted to the frequency domain for computing the velocity.

In terms of performance, PDV has the remarkable ability to measure a broad range of velocities from a few m/s up to roughly $50 \mathrm{~km} / \mathrm{s}$, with high accuracy of $10 \mathrm{~m} / \mathrm{s}$ and time resolution of $10 \mathrm{~ns}$ for long durations[8]. In recent years, PDV has been well developed in aspects of multi-point measurement, precision, range and so on [9][10][11][12] .

The beam of laser light in PDV system is contained entirely within fiber, except the light reflected from the surface of the moving target. For this reason, PDV is regarded as an exceptionally robust system. However, the light beam back reflected from the probe was used as the reference light in the original device [7]. Some types of probe available in our lab cannot meet the 
requirement of back reflection in the measurement. Thus we get the reference light in some other way to make it more applicable.

\section{Experimental Setup}

The improved setup which is called generally applicable photon Doppler velocimetry (GAPDV) is shown in Fig. 1. The source was a $3.5 \mathrm{~mW}$ laser worked at $1310 \mathrm{~nm}$. The light from the laser is split into two beams using the first coupler. One beam of light enters port 1 of an optical circulator and emerges at port 2. Then the light is sent to the surface of a moving target via a single mode fiber probe. The back reflected light collected by the probe enters port 2 and exiting at port 3 . The other beam of light enters the second coupler directly along a reference path. There is sufficient original light sent to coupler 2 to ensure the occurrence of interference. Then these two beams of light mix together and then the interference is detected with a high-speed detector.

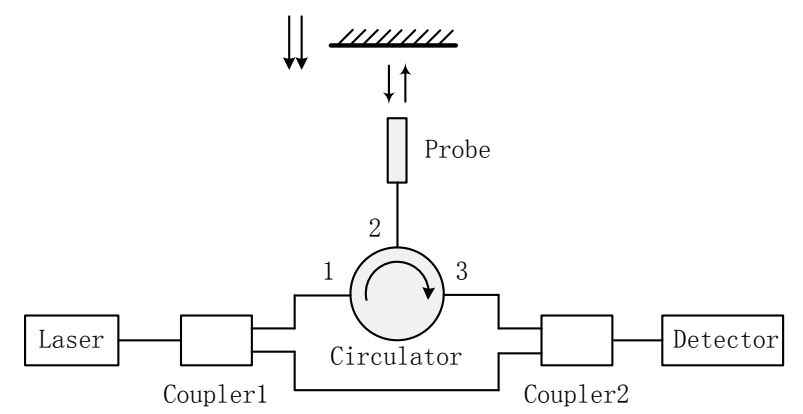

Fig. 1. Experimental system diagram

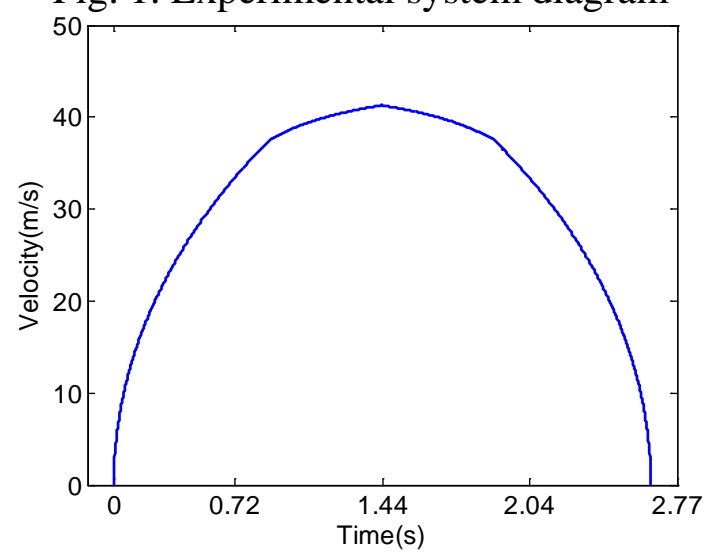

Fig. 2. The simulation velocity of RMS

It is well known that a beat frequency is generated when mixing two sinusoidal wave forms of different frequencies. The interference signal sent to the detector that containing the Doppler shifted light and the original light can be expressed as

$$
E=E_{d} \cos \left(\omega_{d} t+\varphi_{d}\right)+E_{0} \cos \left(\omega_{0} t+\varphi_{0}\right)
$$

where $E_{d}, \omega_{d}, \phi_{d}$ represent for the amplitude, angular frequency and initial phase of the Doppler shifted light separately, $E_{0}, \omega_{0}, \phi_{0}$ represent for the amplitude, angular frequency and initial phase of the original light separately. The intensity of the signal which is proportional to the square of amplitude is given by

$$
\begin{gathered}
E^{2}=E_{d}^{2} \cos ^{2}\left(\omega_{d} t+\varphi_{d}\right)+E_{0}^{2} \cos ^{2}\left(\omega_{0} t+\varphi_{0}\right)+E_{d} E_{0} \cos \left[\left(\omega_{d}+\omega_{0}\right) t+\left(\varphi_{d}+\varphi_{0}\right)\right]+ \\
E_{d} E_{0} \cos \left[\left(\omega_{d}-\omega_{0}\right) t+\left(\varphi_{d}-\varphi_{0}\right)\right]
\end{gathered}
$$

The range of frequency that the detector can respond is between $0 \mathrm{~Hz}-10^{9} \mathrm{~Hz}$, while the frequency of light wave from laser is in the order of $10^{14} \mathrm{~Hz}$. As a result, the frequencies of the terms in the previous formula is too high to the detector except the beat frequency of the last term. The output of the first three terms from the detector is the average of each one. The temporal intensity in the output signal of the detector is given by 


$$
I(t)=I_{d}+I_{0}+\sqrt{I_{d} I_{0}} \cos (2 \pi \Delta f t+\Delta \varphi)
$$

where $I_{d}$ is the intensity of the Doppler shifted light, $I_{o}$ is the intensity of the original light from laser, $\Delta \varphi$ is the relative phase between the Doppler shifted light and the original light.

The basic principle of this setup has no essentially difference compared with Strand's system. Thus it can fit for all the other derivatives based on the original PDV system.

\section{Experiment Results}

For decades, most of experiments testing PDV based setup by using plate impact, gas gun or detonation of explosive device. Several dynamic failure mechanisms can caused dangerous to researchers because of the ejection of fragments. It is already verified that the PDV based systems have the ability to measure a broad range of velocities from a few $\mathrm{m} / \mathrm{s}$ up to several tens of $\mathrm{km} / \mathrm{s}$. Therefore, we used reciprocating motion system (RMS) that is safer to researchers. The moving target in RMS is set on an air bearing linear motion slider with many advantages including zero friction, smooth and silent motion, high speed, and high acceleration and so on. A length of elastic string used in the aviation industry ties to the moving target and stay statically in the middle of the air bearing linear guide rail. At the beginning of the experiment, the moving target is dragged to one end of the rail by a stepping motor. When released, the moving target is ejected towards the other end of the rail. When passing the middle of the rail, the velocity of the moving target reach the maximum. Then the velocity reduces with the reverse force of the elastic string. The moving target does reciprocating motion under the force of the elastic string. Supposing that there is no attenuation in the first a few cycles of the reciprocating motion because of the zero friction of the system. The simulation curve which is corresponding to one of the cycles depicts the movement of the target moving from one end of the rail to the other, and is illustrated in figure 2 . The acceleration reaches the maximum value in the process of the accelerating from $0 \mathrm{~m} / \mathrm{s}$ and the decelerating to $0 \mathrm{~m} / \mathrm{s}$. When the acceleration down to zero, the velocity of moving target can reach roughly $40 \mathrm{~m} / \mathrm{s}$ as shown in the figure.

The graph in figure 3 depicts an example of raw data recorded by the digitizer in an experiment. The range of time in figure 3 corresponds to the last few seconds of the simulation curve in figure 2. The frequency of the raw data is too high to observe the details on this scale. The data of beat frequency was processed by means of STFT method to derive velocity, which is shown in figure 4 . The resolution of time and velocity depends on the length of the STFT chosen by the researcher. Longer windows yield higher velocity resolution but lower time resolution. Shorter windows increase time resolution but decrease velocity resolution. An appropriate length of window is necessary to compute the velocity versus time data. The figure 4 illustrates the process of the movement. The downtrend of the curve in figure 4 is consistent with the simulation curve. However, the curve is abnormal when the velocity fell below roughly several meters per second in all these experiments. It is found that difficulties exist in the research applying PDV based systems [13].

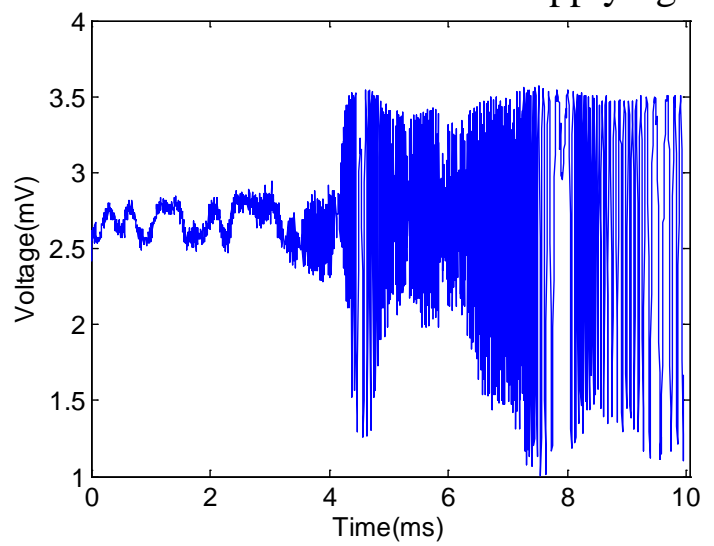

Fig. 3. Raw data from digitizer 


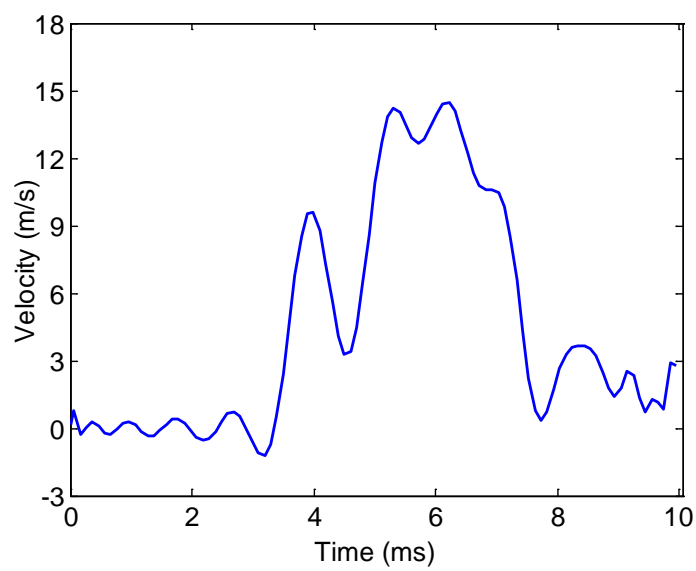

Fig. 4. Data of velocity versus time

\section{Conclusion}

The major difference between the GAPDV system and the conventional PDV system is the source of the non-Doppler shifted light. Specific probe is necessary in conventional PDV system to obtain the appropriate amount of original light. While more types of probe can fit for GAPDV because of the existence of the separate way of the non-Doppler shifted light.

From the data of kinds of PDV systems including GAPDV, we found difficulty in resolving low velocity transients. The improvement of the system is presently under development. The raw data is processed using STFT with fixed windows which is inflexible and hard to choose. Because of this, we are testing some other time-frequency transform methods including wavelet transforms and S-transforms are still under researching.

\section{References}

[1] H. Cummins, N. Knable, L. Gampel, and Y. Yeh, "FREQUENCY SHIFTS IN LIGHT DIFFRACTED BY ULTRASONIC WAVES IN LIQUID MEDIA," Applied Physics Letters, vol. 2, pp. 62-64, 1963.

[2] H. Z. Cummins, N. Knable, and Y. Yeh, "Spurious Harmonic Generation in Optical Heterodyning," Applied Optics, vol. 2, pp. 823-825, 1963/08/01 1963.

[3] Y. Yeh and H. Z. Cummins, "LOCALIZED FLUID FLOW MEASUREMENTS WITH AN He-Ne LASER SPECTROMETER," Applied Physics Letters, vol. 4, pp. 176-178, 1964.

[4] L. M. Barker and R. E. Hollenbach, "Laser interferometer for measuring high velocities of any reflecting surface," Journal of Applied Physics, vol. 43, pp. 4669-4675, 1972.

[5] W. F. Hemsing, "Velocity sensing interferometer (VISAR) modification," Rev Sci Instrum, vol. 50, p. 73, Jan 1979.

[6] L. Levin, D. Tzach, and J. Shamir, "Fiber optic velocity interferometer with very short coherence length light source," Review of Scientific Instruments, vol. 67, pp. 1434-1437, 1996.

[7] O. T. Strand, L. V. Berzins, D. R. Goosman, W. W. Kuhlow, P. D. Sargis, and T. L. Whitworth, "Velocimetry using heterodyne techniques," in 26th International Congress on High Speed Photography and Photonics. vol. 5580, D. L. Paisley, S. Kleinfelder, D. R. Snyder, and B. J. Thompson, Eds., ed Bellingham: Spie-Int Soc Optical Engineering, 2005, pp. 593-599.

[8] B. J. Jensen, D. B. Holtkamp, P. A. Rigg, and D. H. Dolan, "Accuracy limits and window corrections for photon Doppler velocimetry," Journal of Applied Physics, vol. 101, pp. 013523-013523-10, 2007.

[9] D. H. Dolan, R. W. Lemke, R. D. McBride, M. R. Martin, E. Harding, D. G. Dalton, et al., 
"Tracking an imploding cylinder with photonic Doppler velocimetry," Review Of Scientific Instruments, vol. 84, May 2013.

[10]C. H. Cheng, C. W. Lee, T. W. Lin, and F. Y. Lin, "Dual-frequency laser Doppler velocimeter for speckle noise reduction and coherence enhancement," Optics Express, vol. 20, pp. 20255-20265, Aug 2012.

[11]B. C. Frogget, B. M. Cata, B. C. Cox, D. O. DeVore, D. L. Esquibel, D. K. Frayer, et al., "A fisheye lens as a photonic Doppler velocimetry probe," 2012, pp. 84940D-84940D-10.

[12]E. A. Moro and M. E. Briggs, "Note: Simultaneous measurement of transverse speed and axial velocity from a single optical beam," Review of Scientific Instruments, vol. 84, p. 016110, 2013.

[13]T. Ao and D. H. Dolan, "Effect of window reflections on photonic Doppler velocimetry measurements," Review Of Scientific Instruments, vol. 82, Feb 2011. 